\title{
Psychological Assessment of Shyness among Children
}

\author{
Prabhakar, M. L. ${ }^{1}$, Dr. Ravikumar, M. B. ${ }^{2 *}$
}

\section{ABSTRACT}

Shyness is a common but little-understood emotion. Shyness is not a mental disorder. Common sense indicates that Shyness is a behavior pattern characterized by inhibition in some situations. It is a behavior pattern in which the person does not express (or expresses few) thoughts and feelings do not interact actively, and can have physiological alterations like rapid breathing and heart beating. So shyness is the feeling of withdrawal and ineptness when facing situations a person is unfamiliar with. Shyness is a problem for untold numbers of people. The goal of the present study is to explore the shyness of children. Objectives: 1. To study the gender differences in shyness of children. 2. To study the domicile differences in shyness of children. The sample consisted of 120 children; among 60 were male children and 60 were female children. Selected children were measured on Shyness Assessment Test developed by D’Souza (2006). Results indicated that male and female children did not differ in their shyness scores in all the domains-cognitive/affective, physiological, action oriented domains and total shyness scores. Children from rural area were shyer than children from urban areas in all the domainscognitive/affective, physiological, action oriented domains and total shyness scores.

Keywords: Shyness and Children.

Shyness is a common but little-understood emotion. Shyness is not a mental disorder. Common sense indicates that Shyness is a behavior pattern characterized by inhibition in some situations. It is a behavior pattern in which the person does not express (or expresses few) thoughts and feelings do not interact actively, and can have physiological alterations like rapid breathing and heart beating. So shyness is the feeling of withdrawal and ineptness when facing situations a person is unfamiliar with. Shyness is a problem for untold numbers of people. The agony of shyness transcends national boundaries, or divisions of age, sex, race, and nationality. It speaks to fundamental issues around trust, safety, self-esteem and identity, anxiety and fear. Shyness may be defined experientially as discomfort and/or inhibition in interpersonal situations that interferes with pursuing one's interpersonal or professional goals. It is a form of excessive selffocus, a preoccupation with one's thoughts, feelings and physical reactions. It may vary from mild social awkwardness to totally inhibiting social phobia. Shyness may be chronic and dispositional, serving as a personality trait that is central in one's self-definition. Situational shyness involves experiencing the symptoms of shyness in specific social performance situations

\footnotetext{
${ }^{1}$ Counsellor, ICTC, Koppa PHC, Maddur Taluk, Mandya District, Karnataka, India

${ }^{2}$ Counsellor, ART Centre, Mandya Institute of Medical Sciences, Mandya, Karnataka, India *Corresponding Author (C) 2015 I Prabhakar M, Ravikumar M; licensee IJIP. This is an Open Access Research distributed under the terms of the Creative Commons Attribution License (http://creativecommons.org/licenses/by/2.0), which permits unrestricted use, distribution, and reproduction in any Medium, provided the original work is properly cited.
} 


\section{Psychological Assessment of Shyness among Children}

but not incorporating it into one's self-concept. Shyness reactions can occur at any or all of the following levels: cognitive, affective, physiological and behavioral, and may be triggered by a wide variety of arousal cues. Among the most typical are: authorities, one-on-one opposite sex interactions, intimacy, strangers, having to take individuating action in a group setting, and initiating social actions in unstructured, spontaneous behavioral settings. Metaphorically, shyness is a shrinking back from life that weakens the bonds of human connection.

Shyness is difficult to define; researchers use various concepts to explain its many facets. The term shyness was scientifically discussed as early as 1872 when Darwin (1872), wrote, “This odd state of mind, often called shame face redness, or face shame, appears to be one of the most efficient of all the causes of blushing. Darwin $(1872,1999)$ concluded that because a shy individual has a low degree of self-confidence and a high degree of self-consciousness, he or she would naturally fear the presents of strangers (Alm, 2006). Tomkins (1963) defined shyness as an aspect of the underlying fundamental emotion of shame. Zimbardo (1977) indicated that shyness is fuzzy concept, the closer one looks the more varieties that be discovered. No single definition can be adequate because shyness means different things to different people.

Nayyal et al (1996) examined shyness and some dimensions of personality they investigated the development of shyness through different age groups, mainly late childhood until early adolescence. The study also tried to investigate the relationship between shyness and extraversion and neuroticism among Qatari samples. The Arabic scale of shyness had a consistent factorial structure among Qatari samples. The Arabic scale of shyness was administered to 494 Qatari students from different age groups (11-16 years). The 14-year-old age group attained the highest mean scores in the male groups, whereas the 16-year-old age group attained the highest mean scores among the female groups. This was discussed in light of socialization process. The study showed significant correlation between shyness and neuroticism among males and females. For the factorial study, 2 factors have been extracted among the 2 samples of the study.

Pines and Zimbardo (1978) made a comparative study of the personal and cultural dynamics of shyness between Israelis, American Jews and Americans. About 821 Israelis aged 12 years and above were administered a Hebrew translation of a shyness survey that had been used with American Jews and American gentiles by the researchers. Results of the present study were compared with those obtained from the American subjects. The most consistent finding was that Israelis reported less shyness than did Americans. Israelis also tended to be less self-conscious and spent less time in introspection of Israelis. About 79\% of Americans mentioned selfconsciousness and reported extreme awareness of themselves as part of the experience of shyness. The emotional experience that Americans call shyness may not be described as such by Israelis according to the study.

Cheek et al (1999) examined varieties of shyness in adolescence and adulthood such as extreme fear, shyness and social phobia at the stages of adolescence and adulthood. After considering the distinction between early and later-developing shyness, the scholars described the 3- 
component model of adult shyness by introducing a new approach to withdrawn and dependent subtypes of shyness.

Shyness is extremely painful emotionally for a child and can have negative effects in many areas of a child's life. Many shy children develop low self-esteem and may lack self worth. Shy children have difficulty making friends and may be so timid that they will not ask for help from teachers which could set them back in their studies. These negative traits can also follow a child into adolescence and adulthood. Psychologists of varying theoretical persuasions have long held that social experiences are critical to normal developmental trajectories and that the lack of such experiences is worthy of compensatory attention. Surprisingly, however, little empirical attention has been directed to the study of the psychological significance of social solitude for children. Shy children are wary in the face of new social situations and perceived social evaluation. In this regard, the transition to kindergarten may represent a particularly challenging task for shy children. Shyness does influence children's cognitive test performance and its impact is larger when children are tested face-to-face rather than in a more anonymous group setting (Crozier \& Hostettler, 2003).

\section{METHOD}

\section{Objectives}

1. To study the gender differences in shyness of children.

2. To study the domicile differences in shyness of children.

\section{Hypotheses}

H1. Male and female children differ significantly in their shyness scores.

H2. Urban and rural children differ significantly in their shyness scores.

\section{Participants}

The sample consisted of 120 children; among 60 were male and 60 were female children. For sample selection randomly sampling method was used. Their age ranges from 08 to 15 years with the mean age 11.50 years.

Table 1: Distribution of the sample-Gender and Domicile

\begin{tabular}{|c|c|c|c|}
\hline \multirow{2}{*}{ Area } & \multicolumn{2}{|c|}{ Gender } & \multirow{2}{*}{ Total } \\
\cline { 2 - 3 } & Male & Female & \\
\hline Urban & 30 & 30 & 60 \\
\hline Rural & 30 & 30 & 60 \\
\hline Total & 60 & 60 & 120 \\
\hline
\end{tabular}




\section{Tools}

Shyness Assessment Test (SAT): Shyness Assessment Test developed by D’Souza (2006), It consists of 54 items and requires the subject to indicate his/her response by marking Yes, or No. If the answer is 'yes', further, the participant has to indicate one of the three levels-low, medium or high. The items in the test pertain to three domains of shyness- Cognitive/Affective (32 items), Physiological (11 items) and Action oriented (11 items). The reliability index ascertained by split half (odd-even) method and Cronbach's alpha coefficient for the scale as a whole were found to be 0.735 and 0.812 respectively.

\section{Procedure}

The tests were administered to the children in groups of 2-4 subjects per group. Data collection was done in a sessions and session lasted for about 30-40 minutes. In the a session, rapport was established with the children and they were asked to introduce themselves. During the interaction with the teachers active co-operation from the respective class teachers sought to get more conducive environment with the children. The children were then they were administered the Shyness questionnaire. They were given appropriate instructions and the questions were read out to them. They were asked to indicate their responses in the respective sheets given to them. Whenever they had doubt in understanding questions, the test administrator made those questions very clear to them in their local language and helped them to tick or answer at appropriate place with the help their class teacher. Then the data was scored and statistically analysed by using descriptive and test.

\section{RESULTS AND DISCUSSION}

Table 2 : Mean scores of male and female children in different domains of shyness including total shyness and results of Independent sample's 't' test

\begin{tabular}{|c|c|c|c|c|c|}
\hline Domains of shyness & Sex & Mean & Sd & 't' value & $\mathbf{P}$ value \\
\hline \multirow{2}{*}{ Cognitive/affective } & Male & 31.12 & 8.35 & \multirow{2}{*}{.386} & \multirow{2}{*}{.700} \\
\hline & Female & 31.67 & 7.22 & & \\
\hline \multirow{2}{*}{ Physiological } & Male & 9.30 & 4.57 & \multirow[b]{2}{*}{.972} & \multirow{2}{*}{.333} \\
\hline & Female & 8.55 & 3.86 & & \\
\hline \multirow{2}{*}{ Action oriented } & Male & 8.83 & 4.23 & \multirow[b]{2}{*}{.736} & \multirow{2}{*}{.463} \\
\hline & Female & 8.28 & 3.94 & & \\
\hline \multirow{2}{*}{ Total Shyness } & Male & 49.15 & 14.60 & \multirow{2}{*}{.626} & \multirow{2}{*}{.533} \\
\hline & Female & 47.63 & 11.81 & & \\
\hline
\end{tabular}

Table No. 2 shows mean, SD and t values for Cognitive/affective, Physiological, Action oriented and total shyness of children. Cognitive/affective on shyness of male children (Mean= 31.12; 
$\mathrm{SD}=8.35)$ and female children (Mean= 31. 67; $\mathrm{SD}=7.22)(\mathrm{t}=.386 ; \mathrm{p}=.700)$ indicating nonsignificant difference. Male and female children had statistically equal scores in Cognitive/affective on shyness. Physiological on shyness of male children (Mean $=9.30 ; \mathrm{SD}=$ 4.57) and female children (Mean= 8.55; $\mathrm{SD}=3.86)(\mathrm{t}=.972 ; \mathrm{p}=.333)$ indicating non- significant difference. Male and female children had statistically equal scores in Physiological on shyness. Action oriented on shyness of male children (Mean $=8.83 ; \mathrm{SD}=4.23$ ) and female children $(\mathrm{Mean}=8.28 ; \mathrm{SD}=3.94)(\mathrm{t}=.736 ; \mathrm{p}=.463)$ indicating non- significant difference. Male and female children had statistically equal scores in Action oriented on shyness. Total shyness of male children $($ Mean $=49.15 ; \mathrm{SD}=14.60)$ and female children $($ Mean $=47.63 ; \mathrm{SD}=11.81)(\mathrm{t}=$ .626; $\mathrm{p}=.533$ ) indicating non- significant difference. Male and female children had statistically equal scores in total on shyness.

Table 3. Mean scores of children living in urban and rural areas on various domains of shyness and results of independent samples ' $t$ ' test

\begin{tabular}{|c|c|c|c|c|c|}
\hline $\begin{array}{c}\text { Domains of } \\
\text { shyness }\end{array}$ & Area & Mean & SD & 't' value & P value \\
\hline \multirow{2}{*}{$\begin{array}{c}\text { Cognitive/ } \\
\text { affective }\end{array}$} & Urban & 28.90 & 8.15 & \multirow{2}{*}{3.692} & .000 \\
\cline { 2 - 4 } & Rural & 33.88 & 6.55 & & \\
\hline \multirow{2}{*}{ Physiological } & Urban & 7.80 & 4.66 & \multirow{2}{*}{3.014} & \multirow{2}{*}{.003} \\
\cline { 2 - 4 } & Rural & 10.05 & 3.43 & & \\
\hline \multirow{2}{*}{ Action oriented } & Urban & 7.35 & 3.93 & \multirow{2}{*}{3.381} & \multirow{2}{*}{.001} \\
\cline { 2 - 4 } & Rural & 9.77 & 3.90 & \\
\hline \multirow{2}{*}{ Total Shyness } & Urban & 43.30 & 13.48 & \multirow{2}{*}{4.546} & \multirow{2}{*}{.000} \\
\cline { 2 - 4 } & Rural & 53.48 & 10.93 & & \\
\hline
\end{tabular}

Table No. 3 shows mean, SD and t values for Cognitive/affective, Physiological, Action oriented and total shyness of urban and rural children. Cognitive/affective on shyness of urban children (Mean= 28.90; $\mathrm{SD}=8.15)$ and rural children (Mean = 33.88; $\mathrm{SD}=6.55)(\mathrm{t}=3.692 ; \mathrm{p}=.000)$ indicating highly significant difference. Rural children were statistically higher than urban children in Cognitive/affective on shyness. Physiological on shyness of urban children (Mean = 7.80; $\mathrm{SD}=4.66)$ and rural children (Mean= 10.05; $\mathrm{SD}=3.43)(\mathrm{t}=3.014 ; \mathrm{p}=.003)$ indicating highly significant difference. Rural children were statistically higher than urban children in 
physiological on shyness. Action oriented on shyness of urban children (Mean $=7.35$; $\mathrm{SD}=$ 9.77) and rural children (Mean= 9.77; $\mathrm{SD}=3.90)(\mathrm{t}=3.381 ; \mathrm{p}=.001)$ indicating highly significant difference. Rural children were statistically higher than urban children in Action oriented on shyness. Total shyness of urban children (Mean $=43.30 ; \mathrm{SD}=13.48$ ) and rural children (Mean $=53.48 ; \mathrm{SD}=10.93)(\mathrm{t}=4.546 ; \mathrm{p}=.000)$ indicating highly significant difference. Rural children were statistically higher than urban children in overall shyness.

Figure 1. Mean scores of children living in urban and rural areas on various domains of shyness.

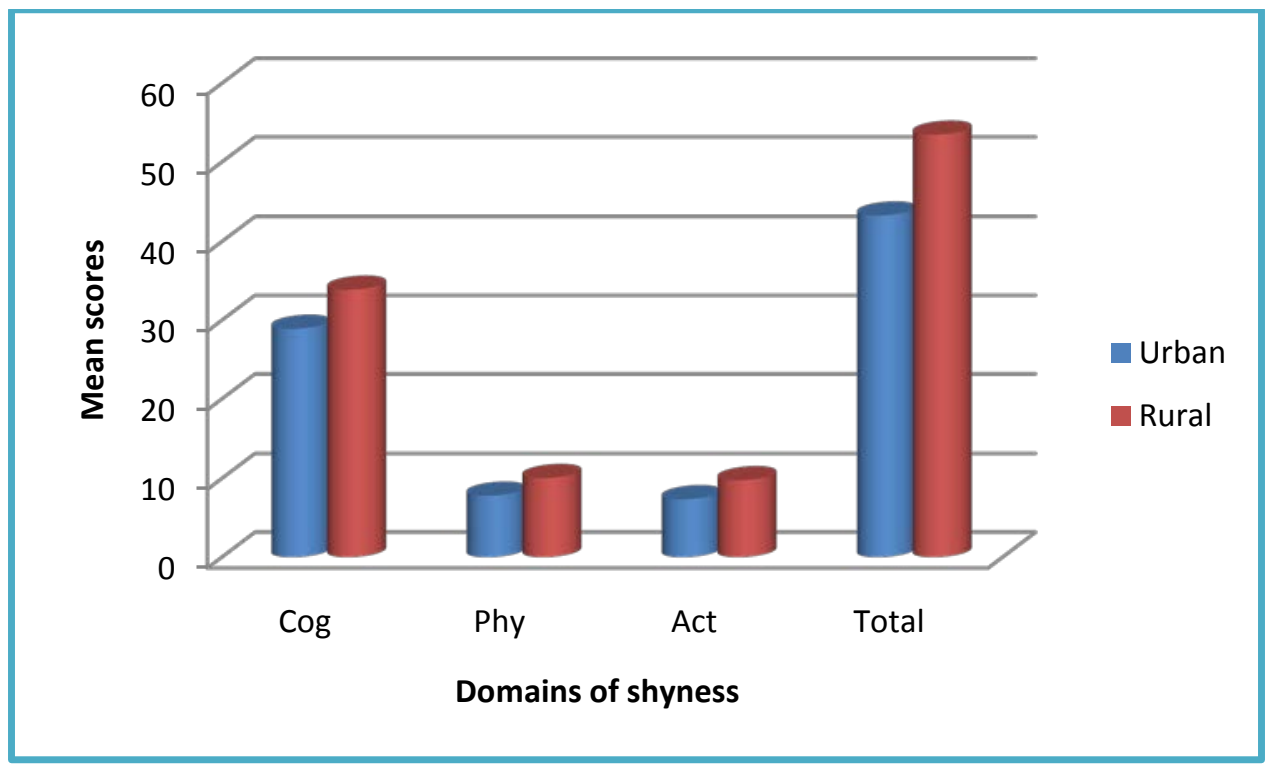

\section{H1. Male and female children differ significantly in their shyness scores.}

Hypothesis 1 is rejected for all the domains- cognitive/affective, physiological, action oriented and total shyness scores. The studies related to gender differences are quite a few and those are summarized. Study by Natesha and D'Souza (2007) reported no gender-wise differences between boys and girls, though in India it is believed that girls express more shyness than boys. Henderson and Zimbardo (1996) also reported no gender difference shyness, but men have typically learned tactics for concealing their shyness because it is considered a feminine trait in most countries. In Mexico males are less likely than females to report shyness.

Hermann \& Betz (2004) showed that American men rated themselves as significantly shyer than did American women. Also, Pilkonis (1977) found that a higher percentage of men than women labeled themselves shy ( 46.4 vs. $33.0 \%$, respectively). Other studies suggest that there are no major differences between males and females regarding either degree of shyness or prevalence of shy vs. non-shy individuals.

Hamer \& Bruch (1994) showed no significant relationship between shyness and gender. In a study of the prevalence of social phobia in a large sample of college students, the distribution of 
males and females in subsamples of highly and moderately shy individuals did not significantly differ (Chavira et al., 2002). Also, when conducting a search for the term 'shyness' in the database PsycINFO (in September 2004), a total of 422 studies conducted on human participants over the age of 18 years were found. Of these, 144 studies (34\%) were conducted on only male participants whereas 155 studies (37\%) were conducted on only female participants. Thus, there seems to be an about equal number of studies conducted on males and females (Alm, 2006).

\section{H2. Urban and rural children differ significantly in their shyness scores.}

Hypothesis 2 is accepted for all the domains and total shyness scores as the obtained statistical values obtained for difference between children from urban and rural areas were found to be highly significant. In other words area had significant influence over shyness. Children in rural areas had higher levels of shyness than urban area children. One of the important aspect to be highlighted that most of the students studying in rural areas are monolinguals and mostly they study in Kannada language, whereas in urban area students are coming from diverse areas and multilingual. Students in rural area studying in Kannada media they may have felt inferior to their counterparts in urban area, which may lead to increased shyness among rural students. Further, students in urban area are exposed to variety of situations - psychological, physiological and cognitive aspects than students studying in rural areas. Study by D’Souza and Urs (2007) reports the prevalence of shyness among children with age ranging from 5-13 years who were studying in and around Mysore city. A total of 1200 children studying in classes from I to VIII from English and Kannada medium were randomly selected for the study. They were administered Shyness scale (Crozier, 1995) in one setting. Chi-square and contingency coefficient tests were applied to see the significance of difference between different levels of shyness and association between gender and age with shyness levels. Results revealed that on the whole $26.2 \%$ of the children showed high levels of shyness, followed by $36.6 \%$ moderate and remaining $37.3 \%$ of the children showed low levels of shyness. Students studying in rural area had significantly higher shyness compared to students studying in urban areas. A startling observation that children from this study (India) showed higher levels of shyness than children of other countries. Of all the age groups children with the age of 11-13 years were found to have higher levels of shyness than children in the age group of 8-10 years. Gender-wise comparison revealed no significant difference between boys and girls. Treatment aspects of shyness are also discussed.

\section{CONCLUSIONS}

1. Male and female children did not differ in their shyness scores in all the domainscognitive/affective, physiological, action oriented domains and total shyness scores.

2. Children from rural area were shyer than children from urban areas in all the domainscognitive/affective, physiological, action oriented domains and total shyness scores. 


\section{REFERENCES}

Alm,C.(2006). The Role of Causal Attribution and Self-Focused Attention for Shyness. LiUTryck, Linkšping.

Chaviraa, D. A., Stein, M.B and Malcarne, V. L. (2002). Scrutinizing the relationship between shyness and social phobia. Journal of Anxiety Disorders.16, 585-598.

Check, J. M., Melchior, L. A. \& Catpentieti, A. M. (1999). Shyness and self-concept. In: L. M. Hattman \& K. R. Blankstein(Eds.), Perception of self in emotional disorder and psychotherapy. New York: Plenum Press.

D’Souza, L. \& Urs, G.B. (2007). Assessment of shyness among adolescent students studying in rural and urban areas. Asia Pacific Review of Rural and Tribal Issues, 1, 10-14.

D’Souza, L. (2006). Shyness assessment test. Mysore: University of Mysore.

Darwin,C. (1999).The expression of emotions in man an animals.(3rd ed.). In: P. Ekman, (Ed.), London, UK: Fontana. (original work published 1872).

Hamer, R. J., \& Bruch, M. A. (1994). The role of shyness and private self-consciousness in identity development. Journal of Research in Personality, 28, 436-452.

Henderson, L., \& Zimbardo,P. G.(1998). Shyness: In Encyclopedia of Mental Health. San Diego, CA: Academic Press.

Nayyel, M.E. (1996). Shyness and some dimensions of personality Drasat Natseyah, 6,173-230.

Pilkonis, P. A. (1977). Shyness, public and private, and its relationship to other measures of social behavior. Journal of Personality, 45, 585-595.

Pines, A., \& Zimbardo, P.G. (1978). The personal and cultural dynamics of shyness A comparison between Israelis, American Jews and Americans. Journal of Psychology \& Judaism, 3, 81-101

Tomoko Hyugano and Takashi Oguchi (2007). The 'situational sense of nigate' by sociometric status and personality traits among elementary school students. The Japanese Journal of Experimental Social Psychology, Vol. 46, (2), 133-142.

Zimbardo, P.G. (1977). Shyness. Reading, MA: Addison-Wesley. 